\title{
Advanced Devices and Systems for Radiation Measurements
}

\author{
prepared by \\ Glenn F. Knoll, Project Director \\ David K. Wehe, Faculty Participant \\ Zhong He, Research Scientist \\ Carla Barrett, Graduate Student Research Assistant \\ Jun Miyamoto, Graduate Student Research Assistant \\ of \\ The University of Michigan \\ College of Engineering \\ Department of Nuclear Engineering \& Radiological Sciences \\ Ann Arbor, Michigan
}

submitted to

U. S. Department of Energy

Nevada Operations Office

Las Vegas, Nevada

June 1996

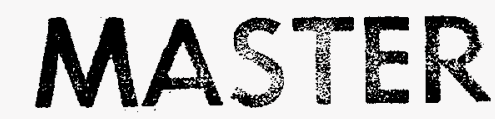

The work upon which this report is based was supported by the U. S. Department of Energy under Contract No. DE-FG08-94NV11630. 


\section{DISCLAIMER}

This report was prepared as an account of work sponsored by an agency of the United States Government. Neither the United States Government nor any agency thereof, nor any of their employees, make any warranty, express or implied, or assumes any legal liability or responsibility for the accuracy, completeness, or usefulness of any information, apparatus, product, or process disclosed, or represents that its use would not infringe privately owned rights. Reference herein to any specific commercial product, process, or service by trade name, trademark, manufacturer, or otherwise does not necessarily constitute or imply its endorsement, recommendation, or favoring by the United States Government or any agency thereof. The views and opinions of authors expressed herein do not necessarily state or reflect those of the United States Government or any agency thereof. 


\section{DISCLAIMER}

Portions of this document may be illegible in electronic image products. Images are produced from the best available original document. 


\section{SINGLE CARRIER SEMICONDUCTOR DETECTOR DEVELOPMENT}

Over the past several months, our work at the University of Michigan has concentrated on developing new techniques to overcome the limitations present in typical room temperature semiconductor materials when incorporated in gamma ray spectrometers. A major problem is the poor mobility of holes that leads to partial charge collection and poor energy resolution when configured as conventional radiation detectors. Many materials also show significant inhomogeneity and corresponding nonuniformity of charge carrier transport.

Our most recent work continues our long-standing efforts to develop semiconductor detectors based on the collection of only a single type of charge carrier. Our best results are an extension of the principle of coplanar electrodes first described by Paul Luke of Lawrence Berkeley Laboratory 18 months ago. This technique, described in our past progress reports, has the effect of deriving an output signal from detectors that depends only on the motion of carriers close to one surface. Since nearly all of these carriers are of one type (electrons) that are attracted to that electrode, the net effect is to nearly eliminate the influence of hole motion on the properties of the output signal. The result is that the much better mobility of electrons in compound semiconductors materials such as CZT can now be exploited without the concurrent penalty of poor hole collection.

We have also developed new techniques in conjunction with the coplanar electrode principle that extends the technique into a new dimension. By proper processing of signals from the opposite electrode (the cathode) from the coplanar surface, we are able to derive a signal that is a good indication of the depth of interaction at which the charge carriers were initially formed. We have been the first 
group to demonstrate this technique, and examples of separate pulse height spectra recorded at a variety of different depths of interaction are shown in several of the figures that follow. Briefly, this technique now provides the opportunity to correct for inhomogeneities as a function of depth into the detector, and also provide us with a new set of data that allows more precise optimization of the overall properties of the device when operated in simple coplanar mode.

Obtaining depth information is one step in the direction of obtaining volumetric point-of-interaction information from the detector. If one could know the coordinates of each specific interaction, then corrections could be applied to account for the inhomogeneities that currently plague many room-temperature devices. Although extracting additional position coordinates adds to the complexity of the system, advances in multi-channel readout electronics over the past several years now makes such an approach appear to be technology feasible. The coplanar electrode approach already subdivides the detector into strips, and an additional coordinate could be obtained simply by processing the signals from each strip in a separate channel. Although we have not yet demonstrated position sensing beyond that of depth, we feel that this general approach is the correct one to ultimately overcome the limitations now set by materials properties in commercially available room-temperature semiconductor materials. We are confident that recent advances in multi-channel chip electronics (128 linear channels on a single chip are now commercially available) will permit rapid advances in this direction.

\section{RECENT RESULTS}

We have obtained a $1 \mathrm{~cm}^{3}$ cubic crystal of CZT and had coplanar electrodes fabricated on one surface. Using the conventional coplanar electrode technique, an 
energy resolution of $1.8 \%$ FWHM at $662 \mathrm{keV}$ was obtained without any selection of the gamma ray events that were accepted. When we plotted the energy spectra as a function of the gamma ray interaction depth, energy resolution of about 1.5\% FWHM was obtained over about two-thirds of the detector volume. We have achieved the goal we set last year of improving the energy resolution to the $1-2 \%$ range, and done so on a larger crystal than we have previously used. To our knowledge, the results quoted above and shown in the figure that follows are the best obtained by any group with a room-temperature CZT detector of this volume. Since the results are still very new, we feel that the potential exists for improving performance even further with additional development work.

We have also set up a system to study the digitized pulse waveforms from our detectors. This system allows insights to be gained as to the detailed mechanisms that are occurring within the detector, and has been a great help in optimizing factors such as electrode asymmetry effects and crystal inhomogenity. It also allows us to optimize the techniques used for pulse shaping and processing.

Although nearly all of our work is currently with cadmium zinc telluride (CZT), we are also exploring the possibility of applying similar techniques to mercuric iodide detectors. It is well known that major limitations in mercuric iodide performance are related to striations and other inhomogeneities in the crystals that are grown. Since our long-term objective is to obtain volumetric information from materials, we feel that there is some prospect of greatly improving the performance of mercuric iodide spectrometers by taking into account the nonuniformities and making appropriate corrections. We are in the process of working with a supplier of mercuric iodide crystals and specifying coplanar electrode patterns that will be fabricated on their surfaces. We would like to 
investigate whether the gains that have been realized in CZT are also applicable to mercuric iodide when coplanar electrode techniques are applied.

\section{DIRECTIONAL GAMMA RAY DETECTOR}

We are continuing to carry out modeling studies of different concepts aimed at providing coarse directionality to portable gamma ray detector systems. We have investigated techniques that are based on sensing fluorescent $x$-rays emitted from a cubic volume on which detectors are face-mounted, and also are investigating schemes based on the emission of secondary electrons from surfaces. This effort is at a low level (one precandidate doctoral student) and is only at the computational modeling stage at present. If we are able to identify a promising geometry that combines the desired directionality with adequate detection efficiency, we will proceed to bench top testing of small-scale prototypes. None of the schemes examined to date provide enough sensitivity to warrant experimental development at the present time, but we are continuing to explore unique geometries and detection mechanisms to enhance sensitivity while maintaining the directional characteristics of a readily portable device. 


\section{FIGURES 1 AND 2}

The following figures show the results obtained using CZT detectors and the coplanar electrode technique. At the top of the page is the overall gamma ray spectrum using the entire volume of the crystal. At the bottom are separate pulse height spectra extracted for each depth of interaction using techniques described in the text. Results are presented both for a $5 \mathrm{~mm}$ cube (Fig. 1) and a $1 \mathrm{~cm}$ cube. (Fig. 2). All results were obtained at room temperature.

\section{DISCLAIMER}

This report was prepared as an account of work sponsored by an agency of the United States Government. Neither the United States Government nor any agency thereof, nor any of their employees, makes any warranty, express or implied, or assumes any legal liability or responsibility for the accuracy, completeness, or usefulness of any information, apparatus, product, or process disclosed, or represents that its use would not infringe privately owned rights. Reference herein to any specific commercial product, process, or service by trade name, trademark, manufacturer, or otherwise does not necessarily constitute or imply its endorsement, recommendation, or favoring by the United States Government or any agency thereof. The views and opinions of authors expressed herein do not necessarily state or reflect those of the United States Government or any agency thereof. 
Energy spectra of a $5 \mathrm{~mm}$ cube CdZnTe detector

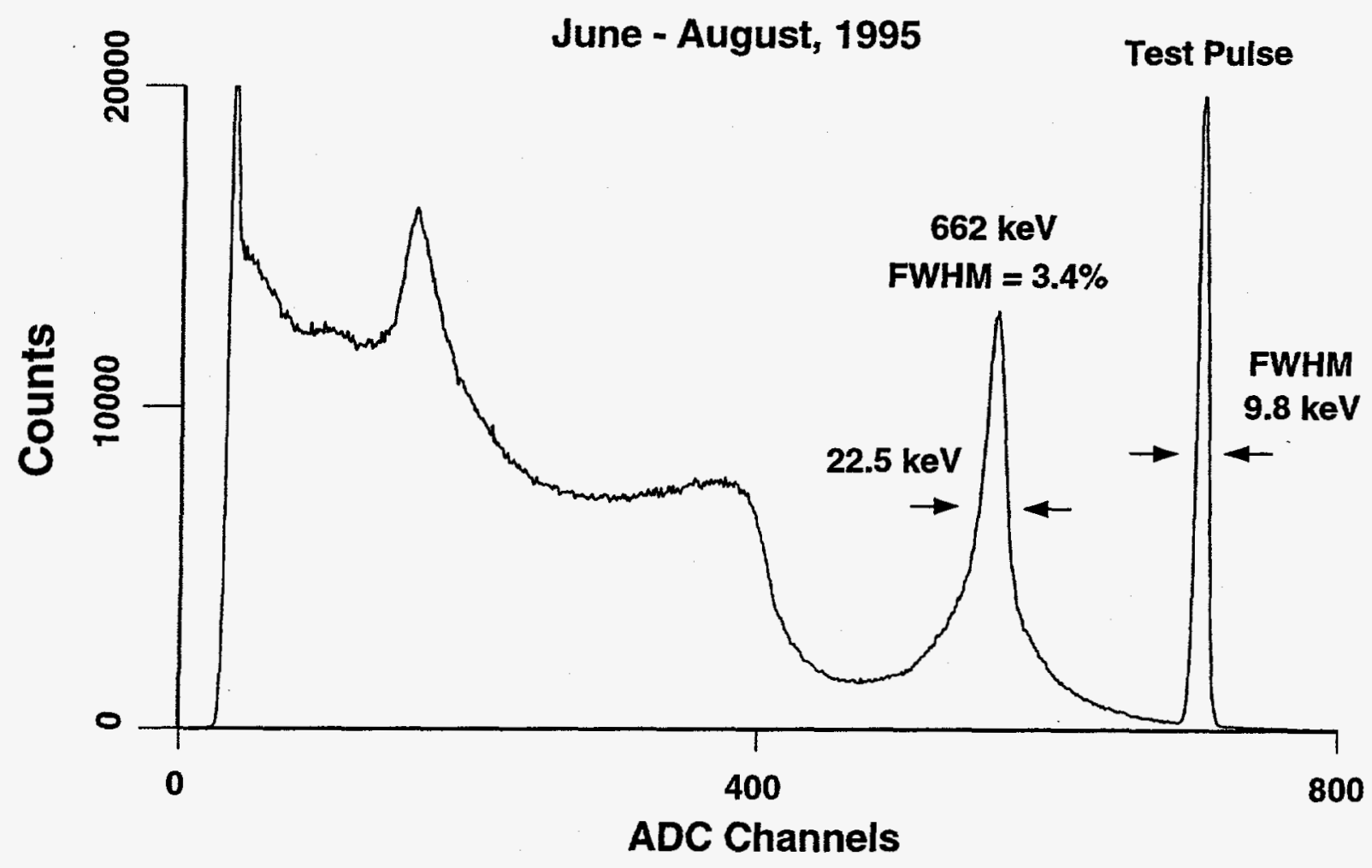

(a) Energy spectrum from all detector volume

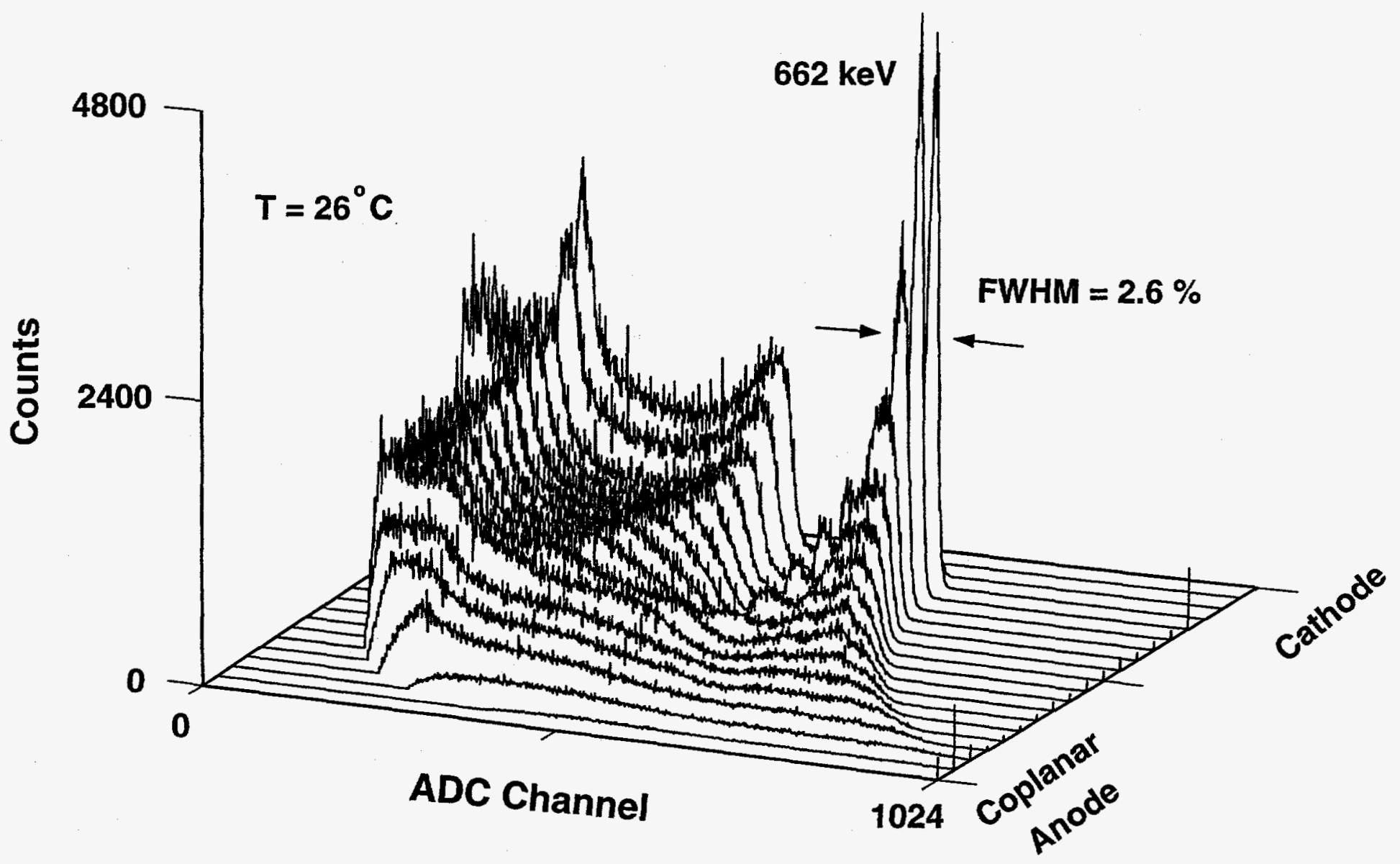

(b) Energy spectra vs interaction depth 
Energy spectra of a $1 \mathrm{~cm}$ cube coplanar CZT detector $\left(\mathrm{V}(\mathrm{c}-\mathrm{a})=-1400 \mathrm{~V}, \mathrm{~V}(\mathrm{a} 1-\mathrm{a} 2)=+30 \mathrm{~V}, \mathrm{~T}=22.5^{\circ} \mathrm{C}\right.$, shaping time $=2$ us)
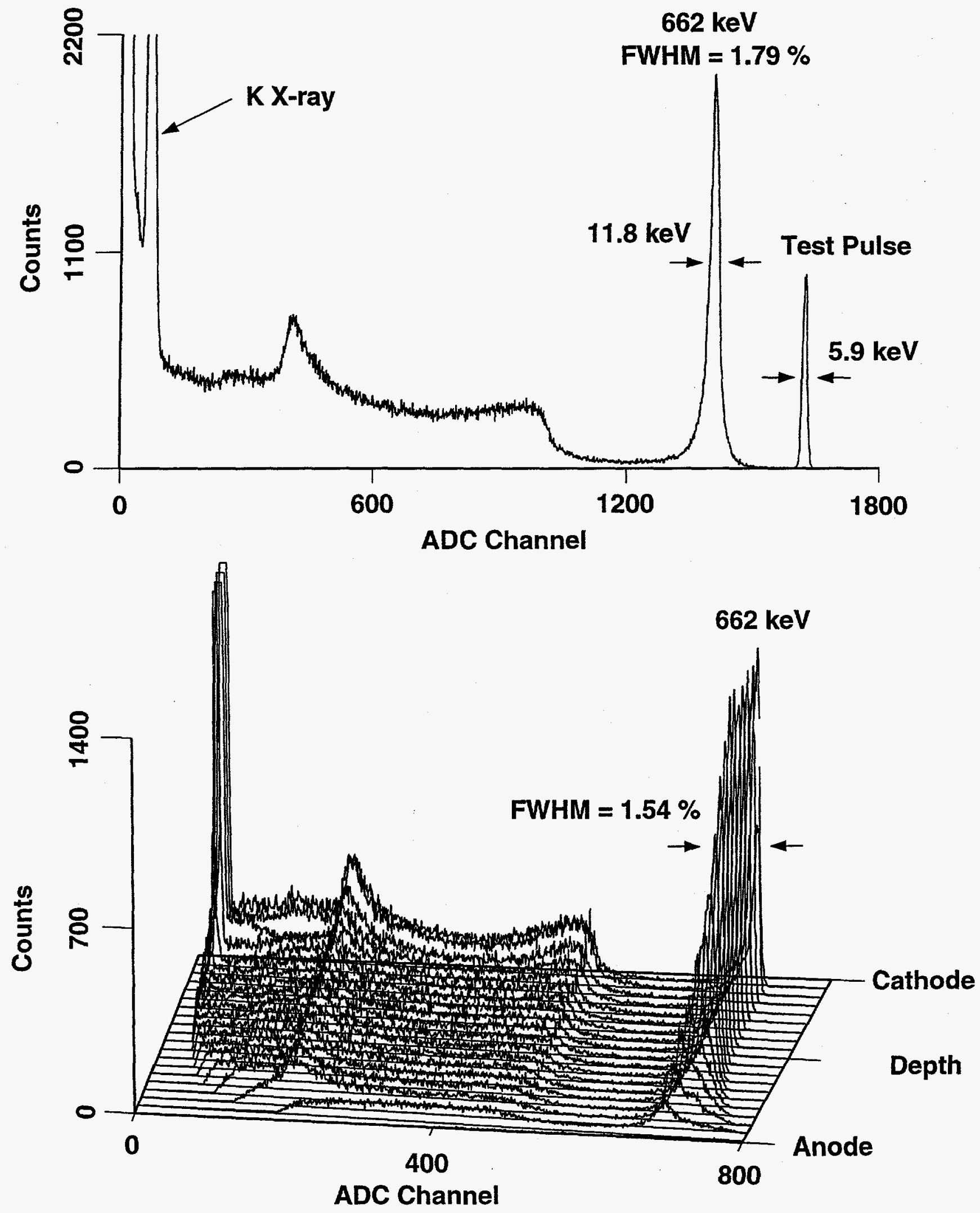

Z. He, 1996-4-23

Data file $=$ p301450e.txt \& p301450a.dat 


\section{APPENDIX A}

The following paper was submitted in April for inclusion on the program of the 1996 Nuclear Science Symposium to be held in San Diego this fall. It describes some results that have now been improved upon in our more recent work. We will present updated data at the time of the meeting. 


\title{
Position Sensitive Single Carrier CZT Detectors
}

\author{
Z. He, G.F. Knoll, D.K. Wehe, J. Miyamoto \\ Department of Nuclear Engineering and Radiological Sciences, \\ University of Michigan, Ann Arbor, MI 48109
}

The single polarity charge sensing method has been studied using coplanar electrodes on CdZnTe gamma-ray detectors. This method can ameliorate the hole trapping problem of room-temperature semiconductor detectors. Our earlier measurement results confirmed that the energy resolution can be dramatically improved compared with that obtained using the conventional read out method on $5 \mathrm{~mm}$ cube CZT detectors. This paper presents our progress on CZT detectors of larger volume, such as $1 \mathrm{~cm}$ cubes. We suggested a method to obtain gamma-ray interaction depth between the cathode and the anode and our latest investigation is reported here. This technique can be used to correct for the electron trapping as a function of distance from the anodes. Significant improvement in energy resolution is demonstrated on corrected energy spectra by using a linear compensation technique and a method using a depth-dependent correction factor, and the two techniques are evaluated. Experimental results show that a position resolution of about $1 \mathrm{~mm}$ FWHM at $122 \mathrm{keV}$ can be obtained on a 1 $\mathrm{cm}$ cube CZT detector. These results will be of interest in the design of higher performance and imaging related room-temperature semiconductor gamma-ray detectors.

\section{For: 1996 IEEE Nuclear Science Symposium}

1. Topics: Solid State Devices.

2. Oral/Poster

3. Corresponding author: Zhong He

Department of Nuclear Engineering and Radiological Sciences, University of Michigan,

Ann Arbor, MI 48109-2104.

Tel: (313)764-5285

Fax: (313)763-4540

E-mail: hezhong@engin.umich.edu 


\title{
Position Sensitive Single Carrier CZT Detectors
}

\author{
Z. He, G.F. Knoll, D.K. Wehe, J. Miyamoto \\ Department of Nuclear Engineering and Radiological Sciences, \\ University of Michigan, Ann Arbor, MI 48109
}

\begin{abstract}
Summary
Room-temperature semiconductor $\gamma$-ray detectors having high atomic numbers and wide band gaps have long been under development because of their high stopping power and freedom from cryogenic cooling. $\mathrm{HgI}_{2}, \mathrm{CdTe}$ and $\mathrm{CdZnTe}$ detectors appear to have the most promise. However, the energy resolution achieved using conventional planar electrodes is poor predominatly due to charge trapping and polarization effects which have limited the detector performance. Luke [1] proposed a coplanar electrode structure on semiconductor $\gamma$-ray detectors somewhat analogous to the Frisch grid commonly employed in gas-filled ion chambers. The coplanar electrodes collect electron carrier signal only, therefore, the hole trapping problem can be eliminated. This method has been demonstrated to be able to improve $\gamma$-ray energy resolution significantly. Subsequently, Barrett et al. [2] showed that the same principle can be applied on 2-D pixulated pad detectors, and $\mathrm{He}$ [3] derived the analytical approximation of the potential distribution for some configurations of coplanar electrodes commonly used, and proposed [4] a method to make a semiconductor detector to be position sensitive to the $\gamma$-ray interaction depth between the cathode and coplanar anodes. An energy resolution of $3.4 \%$ FWHM at $662 \mathrm{keV}$ and a position resolution of $\sim 0.9 \mathrm{~mm}$ FWHM were demonstrated on a 5 $\mathrm{mm}$ cube of CZT in our earlier work [4].

This paper reports our latest progress on CZT detectors of larger volume, such as $1 \mathrm{~cm}$ cubes. The position sensing technique has been used to correct for the electron trapping as a function of distance from the anodes. A significantly improved energy resolution of $2.3 \%$ FWHM at $662 \mathrm{keV}$ has been obtained on a $1 \mathrm{~cm}$ cube CZT detector, and the energy spectrum is shown in Fig.1. Furthermore, when a linear compensation technique and a method which uses a depth-dependent correction factor are used to correct for the raw energy spectrum, better energy resolutions are obtained. Position sensitivity is also evaluated on the $1 \mathrm{~cm}$ cube CZT detector and the experimental results show that a position resolution of about $1 \mathrm{~mm}$ FWHM at $122 \mathrm{keV}$ can be obtained.

These results will be of interest in the design of higher performance and imaging-related room-temperature semiconductor $\gamma$-ray detectors.
\end{abstract}

\section{References}

[1] P. Luke "Unipolar Charge Sensing with Coplanar Electrodes - Application to Semiconductor Detectors" IEEE Trans. on Nucl. Sci., Vol.42(4), p.207, 1995.

[2] H.H. Barrett et al. "Charge Transport in Arrays of Semiconductor Gamma-Ray Detectors" Phys. Rev. Let., Vol.75, No.1, p.156, 1995. 


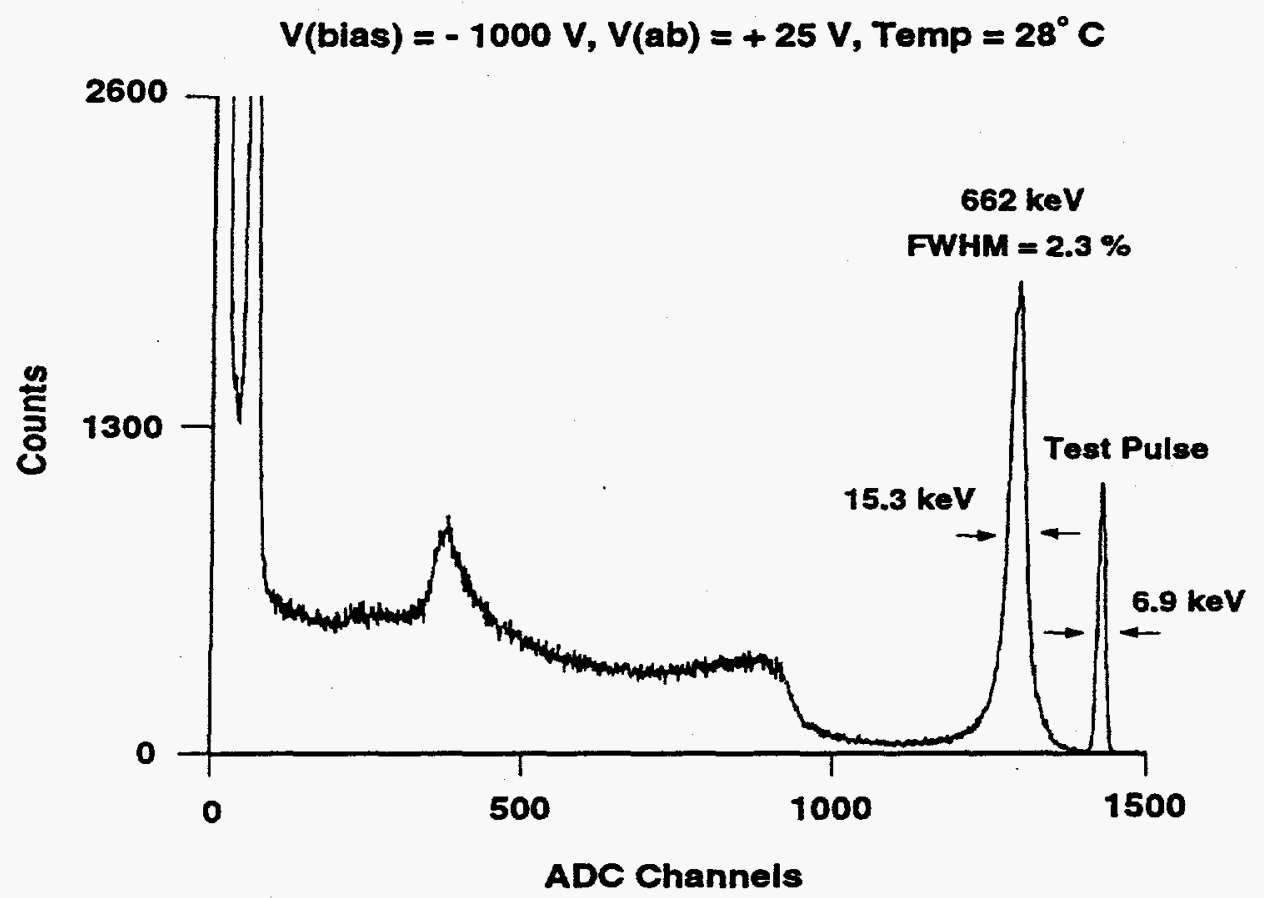

Figure 1: Energy spectrum from a $1 \mathrm{~cm}$ cube CZT detector.

[3] Z. He "Potential Distribution within Semiconductor Detectors Using Coplanar Electrodes" Nuciear Instruments and Methods, A365, p.572, 1995.

[4] Z. He et al. "1-D Position Sensitive Single Carrier Semiconductor Detectors" To appear in: Nuclear Instruments and Methods, 1996.

\section{For: 1996 IEEE Nuclear Science Symposium}

1. Topics: Solid State Devices.

2. Oral/Poster

3. Corresponding author: Zhong He

Department of Nuclear Engineering and Radiological Science, University of Michigan,

Ann Arbor, MI 48109-2104.

Tel: (313)764-5285

Fax: (313)763-4540

E-mail: hezhong@engin.umich.edu 